\title{
APLICAÇÃO DA VERSÃO BRASILEIRA DA ESCALA DE AVALIAÇÃO CLÍNICA DA DEMÊNCIA (CLINICAL DEMENTIA RATING - CDR) EM AMOSTRAS DE PACIENTES COM DEMÊNCIA
}

\author{
Alberto Luiz Grigoli Maia', Cláudia Godinho', Eduardo Daura Ferreira', \\ Vanessa Almeida', Artur Schuh ${ }^{1}$, Jeffrey Kaye'2, Márcia Lorena Fagundes Chaves ${ }^{1}$
}

\begin{abstract}
RESUMO - Objetivo: Avaliar a concordância da escala CDR com critérios diagnósticos e mini exame do estado mental (MEEM), e correlação com escala de Blessed, numa amostra de pacientes do sul do Brasil. Método: A escala foi avaliada em 269 pacientes com doença de Alzheimer (DA), demência vascular e demência questionável num desenho transversal. Os critérios do NINCDS-ADRDA para provável DA e NINDS-AIREN para p rovável demência vascular foram os padrões-ouro. O MEEM, a escala Blessed para gravidade da demência, o escore isquêmico de Hachinski, e uma bateria de testes cognitivos também foram aplicados. Resultados: A concordância com o padrão-ouro foi boa (kappa=0,73), e com o MEEM em categorias foi moderada (kappa= $0,53)$. Observou-se correlação significativa da escala CDR com Blessed $(r=0,96 ; p=0,001)$. Não se observou dife rença de escolaridade ou de idade entre as categorias da escala CDR. Conclusão: A concordância da CDR foi boa para os critérios diagnósticos e moderada para o MEEM. A escala mostrou validade de construto para gravidade de demência. Não se observou impacto da escolaridade sobre este instrumento.
\end{abstract}

PALAVRAS-CHAVE: demência, escala CDR, validação, Mini Exame do Estado Mental, Brasil.

\begin{abstract}
Application of the Brazilian version of the CDR scale in samples of dementia patients
ABSTRACT - Objective: The objective of the study was the analysis of agreement between the CDR scale with diagnostic criteria and mini mental state examination (MMSE), as well as correlation with Blessed scale, in a sample of Southern Brazilian patients. Method: The CDR scale was cross-sectionaly evaluated in 269 dementia patients Alzheimer's disease (AD) vascular dementia, and questionable. The NINCDSADRDA criteria for probable AD and the NINDS-AIREN for probable vascular dementia were the gold standard. The MMSE, the Blessed scale, the Hachinski ischemic score, and a battery of cognitive tests were also applied. Results: The agreement to gold standard was good (kappa=0.73), while to MMSE categorized was moderate (kappa=0.53). A significant correlation with the Blessed scale $(r=0.96 ; p=0.001)$ was observed. Education and age were similar among CDR categories. Conclusion: The global score agreement of the CDR scale with the gold standard was good, and with the MMSE was moderate. We also observed face validity for dementia severity. No impact of education was observed upon CDR global scores.
\end{abstract}

KEY WORDS: dementia, CDR scale, agreement analysis, Mini Mental State, Brazil

A escala de avaliação clínica da demência (clinical dementia rating - (DR) foi desenvolvida em 1979 no p rojeto "Memory and Aging" da Universidade Washington, St. Louis, Missouri, EUA, para graduar demência especialmente na doença de Alzheimer (DA)'. Inicialmente, o objetivo era usar informação de fonte colateral (familiar/cuidador) associada a informação obtida com o próprio paciente durante a anamnese, auxiliadas por alguns instrumentos como a escala Bles- sed para gravidade de demência ${ }^{2}$ e o Mini Exame do Estado Mental (MEEM) ${ }^{3}$. Posteriormente, foi necessário desenvolver a entrevista semi-estruturada para incrementar a confiabilidade da escala e mais recentemente foi colocado um treinamento online (Brief Reliability and Training Protocol - BRT P) também da Universidade Washington ${ }^{4}$. Através deste mesmo local de acesso na Internet, também está disponível um algoritmo para definir o escore global da escala CDR.

\footnotetext{
${ }^{1}$ Ambulatório de Neurogeriatria e Centro de Referência de Doença de Alzheimer, Serviço de Neurologia e Departamento de Medicina Interna, Faculdade de Medicina da Universidade Federal do Rio Grande do Sul(UFRGS), Porto Alegre, Brasil; ${ }^{2}$ Layton Aging and Alzheimer's Disease Center, Oregon Health \& Science University, Portland Oregon USA.
}

Recebido 6 Outubro 2005, recebido na forma final 16 Janeiro 2006. Aceito 17 Março 2006.

Dr. Marcia L. F. Chaves - Rua Ramiro Barcelos 2350 / 2040 - 90035-003 Porto Alegre RS- Brasil. E-mail: mchaves@hcpa.ufrgs.br 
Ao longo dos anos a partir da publicação inicial desta escala houve uma evolução em relação aos resultados de concordância e confiabilidade, partindo de dados sugerindo alta concordância após treinamento de diferentes profissionais em alguns centro $s^{5}$ até os mais recentes que apresentam as dificuldades diferenciais entre aplicadores inexperientes e experientes $^{6}$. A confiabilidade global inter-avaliador da CDR foi 0,62 num ensaio multicêntrico cuja medida de desfecho era o coeficiente kappa. Dentro dos domínios da CDR, os valores de kappa variaram de 0,33 a 0,88 . Este estudo sustenta confiabilidade global moderada a alta, mas demonstra algumas dificuldades importantes na avaliação de demência nas fases mais iniciais ${ }^{7}$. Avaliadores, cegos para diagnóstico, apresentaram concordância de $87 \%$. A concordância observada variou de boa (kappa $=0,66)$ nas dimensões orientação e julgamento \& solução de problemas a excelente $(\mathrm{kappa}=0,83)$ no escore global ${ }^{8}$. A escala CDR já foi traduzida para o japonês, chinês, holandês, belga, inglês da Inglaterra e Austrália, polonês, sueco, espanhol da Espanha e Argentina, e português de Portugal $^{9}$. No Brasil esta escala é ainda muito pouco utilizada, apesar de fazer parte do protocolo do Ministério da Saúde para dispensação das medicações especiais para doença de Alzheimer (MS-PCDT) ${ }^{10}$.

O objetivo deste estudo foi avaliar o desempenho da escala CDR no diagnóstico de demência (vascular e DA), comparando com os critérios NINCDS-ADRDA e NINDS-AIREN e o MEEM, numa amostra de pacientes brasileiros de um ambulatório para demências de um hospital universitário do sul do Brasil.Verificar diferenças de idade e escolaridade entre as categorias da CDR e correlacioná-la com a escala de Blessed também foram propostas do estudo.

\section{MÉTODO}

A avaliação da versão no português brasileiro da escala CDR (com entrevista semi-estruturada) foi realizada em 269 pacientes com DA e demência vascular (incluindo demência questionável) num desenho transversal. Os critérios do DSMIV $^{11}$ para demência, os do NINCDS-ADRDA ${ }^{12}$ para provável DA e NINDS-AIREN para provável demência vascular ${ }^{13}$ foram utilizados. O escore isquêmico de Hachinski ${ }^{14}$ foi também sempre utilizado. As escalas $A D L$ e IADL ${ }^{15}$ e uma bateria de testes cognitivos ${ }^{16}$ fazem parte do protocolo padrão do ambulatório e foram aplicados a todos os participantes do estudo. A bateria de testes cognitivos inclui a extensão de dígitos (ordem direta), extensão de palavras (evocação simples de uma lista de palavras sem conteúdo afetivo), extensão de reconhecimento visual, memória lógica de Wechsler I e II (imediata e tardia) - pequena estória, desenho do relógio, desenho da casa, testes de abstração, cálculo, teste das faces famosas, e praxias ${ }^{16}$.
Um dos autores (MLFC) foi treinado no Alzheimer's Disease and Aging Center da Oregon Health Sciences University, Portland, Oregon (EUA) para utilização da escala CDR e posteriormente treinou a equipe do estudo.

A amostra foi constituída de 121 (45\%) pacientes com p rovável DA, 113 (42\%) pacientes com provável demência vascular, e 35 (13\%) com demência questionável (Tabela 1). Este tamanho amostral é suficiente para detectar uma concordância de pelo menos $80 \%$, com um erro de $10 \%$, e um intervalo de confiança de $95 \%$.

Demência questionável foi definida como (1) comprometimento cognitivo insuficiente para o diagnóstico de demência; (2) comprometimento cognitivo suficiente, mas com prometimento funcional insuficiente para demência; e (3) não preenchimento dos critérios diagnósticos para DA ou vascular. O diagnóstico de demência questionável foi baseado no comprometimento cognitivo através de todos os domínios e não limitado apenas à memória.

A escala CDR foi aplicada de forma independente, seguindo as orientações de Morris ${ }^{17}$. O MEEM ${ }^{3,16}$ e a escala de Blessed para gravidade da demência 2 foram aplicados por outroentrevistador, que era mantido "cego" para os demais resultados.

Tabela 1. Características demográficas e clínicas da amostra.

\begin{tabular}{|c|c|c|}
\hline \multirow{2}{*}{$\frac{\text { Característica }}{\text { Dados categóricos }}$} & \multicolumn{2}{|c|}{ Distribuição } \\
\hline & $\mathrm{N}$ & $(\%)$ \\
\hline \multicolumn{3}{|c|}{ Grupos Diagnósticos } \\
\hline DA & 121 & $(45 \%)$ \\
\hline DV & 113 & $(42 \%)$ \\
\hline DQ & 35 & $(13 \%)$ \\
\hline \multicolumn{3}{|l|}{ Sexo } \\
\hline Homens & 114 & $(42 \%)$ \\
\hline \multicolumn{3}{|l|}{ CDR } \\
\hline 0,5 & 55 & $(20 \%)$ \\
\hline 1 & 69 & \\
\hline 2 & 84 & $(77 \%)$ \\
\hline 3 & 49 & \\
\hline na & 9 & $(3 \%)$ \\
\hline Dados quantitativos & média $\pm \mathrm{DP}$ & \\
\hline Idade & $70,8 \pm 10,0$ & \\
\hline Educação* & $4,8 \pm 3,8$ & \\
\hline Hachinski & $3,9 \pm 3,0$ & \\
\hline MEEM & $14,8 \pm 7,6$ & \\
\hline Blessed & $12,5 \pm 7,1$ & \\
\hline
\end{tabular}

*média de anos completos; na, não aplicado; DA, doença de Alzheimer; DV, demência vascular; DQ, demência questionável; DP, desvio padrão. 
Tabela 2. Distribuição das categorias da escala CDR (reagrupadas em 0,5: demência questionável; e 1,0: demência) de acordo com os critérios diagnósticos (padrão ouro) - N (\%).

\begin{tabular}{ccccc}
\hline & & Questionável & Demência & Total \\
\hline CDR & 0,5 & 23 & 32 & 55 \\
& & $(70 \%)$ & $(14 \%)$ & $(21 \%)$ \\
& 1,0 & 10 & 195 & 205 \\
& & $(30 \%)$ & $(86 \%)$ & $(79 \%)$ \\
& Total & 33 & 227 & 260 \\
\hline
\end{tabular}

Para permitir a análise de concordância de categorias entre a escala CDR e o MEEM utilizamos os seguintes pontos de corte para o MEEM: $<10$, comprometimento grave; 10 15 , comprometimento moderado; entre 15 e os pontos de corte para déficit cognitivo (escolaridade $>4$ anos, MEEM $\leq 24$; escolaridade $\leq 4, \operatorname{MEEM} \leq 17$ ), comprometimento leve; e acima dos pontos de corte, demência questionável. Em função dos pacientes analfabetos e de escolaridade muito baixa (1 e 2 anos) não usamos o tradicional escore 12 para corte de gravidade ${ }^{17}$, pois a diferença entre o ponto de corte de detecção de déficit cognitivo de 17 e o escore 12 para graduar demência grave seriam muito próximos para incluir as categorias de demência leve e moderada.

O estudo foi aprovado pelo Comitê de Ética em Pesquisa do Hospital de Clínicas de Porto Alegre e todos participantes e/ou seus responsáveis deram seu consentimento para participar do estudo.

Análise estatística - A freqüência das variáveis paramétricas é apresentada em média \pm DP e das variáveis categóricas em freqüência absoluta e relativa. Analisamos dados paramétricos com teste $t$ de Student ou ANOVA de 1-via com Tukey post hoc, e testes não paramétricos (Mann-Whitney ou Kruskal-Wallis) para dados ordinais e aqueles que não apresentaram distribuição normal. Variáveis categóricas foram analisadas com testes de associação (qui-quadrado com correção de Yates ou Fisher quando necessários). Correlações não paramétricas (Spearman) foram utilizadas. Confiabilidade diagnóstica foi demonstrada por medidas de concordância dos avaliadores em relação ao padrão-ouro (coeficiente kappa e Kendall tau-b). O programa estatístico SPSS para Windows versão 11.02 foi utilizado para as análises.

\section{RESULTADOS}

A distribuiç̧ão das categorias da escala CDR foi $20 \%$ de casos questionáveis ( $\mathrm{N}=55$ ), e $80 \%$ com demência ( $\mathrm{N}=205)$, sendo que dos demenciados, $34 \%$ leves, $41 \%$ moderados e $25 \%$ graves. A distribuição das categorias da escala CDR, reagrupadas em casos questionáveis $(0,5)$ e demência $(1,0)$ de acordo com os critérios diagnósticos (padrão-ouro), está apresentada na Tabela 2. Observou-se associação significativa
Tabela 3. Distribuição das categorias da escala CDR por cate gorias do Mini Exame do Estado Mental (MEEM).

\begin{tabular}{ccccccc}
\hline & \multicolumn{5}{c}{ Categorias do MEEM } \\
\cline { 3 - 7 } & & $>$ PC & PC-15 & $15-10$ & $<10$ & Total \\
\hline CDR & 0,5 & 35 & 15 & 4 & 1 & 55 \\
& 1,0 & 24 & 26 & 14 & 5 & 69 \\
& 2,0 & 2 & 23 & 26 & 34 & 85 \\
& 3,0 & 2 & 3 & 6 & 34 & 45 \\
& & & & & & \\
& Total & 63 & 67 & 50 & 74 & 254 \\
\hline
\end{tabular}

$\mathrm{PC}$, ponto de corte.

Tabela 4. Média $\pm D P$ da idade e escolaridade nas categorias da $C D R$.

\begin{tabular}{ccccc}
\hline & & $\mathrm{N}$ & Idade * & Escolaridade ** \\
\hline CDR & 0,5 & 55 & $68,3 \pm 10,9$ & $5,24 \pm 4,19$ \\
& 1,0 & 69 & $70,1 \pm 9,7$ & $4,45 \pm 4,21$ \\
& 2,0 & 83 & $72,8 \pm 9,4$ & $4,77 \pm 3,66$ \\
& 3,0 & 49 & $72,2 \pm 9,5$ & $5,02 \pm 3,71$ \\
\hline
\end{tabular}

* $F=1,09 ; p=0,064 * * F=0,44 ; p=0,726$ (ANOVA de 1-via).

entre as categorias diagnósticas do padrão-ouro e da escala CDR (qui-quadrado $=53,4 ; p=0,001$; e razão de probabilidade $=43,18 ; p=0,001)$. A análise de conco rdância mostrou coeficiente kappa=0,73 $(p=0,001)$.

Os escores da escala de Blessed variaram de 3 a 26, com média $12,5 \pm 7,1$ e mediana 11 (interquartil $6,00-19,00)$. O MEEM mostrou escores que variaram de 0 a 29, com média 14,81 $\pm 7,67$, mediana 16 (interquartíl 10,00-21,00). A comparação não-paramétrica dos escores do MEEM entre as categorias da CDR mostrou diferença significativa do ranking das medianas entre todas as categorias (Kruskal-Wallis, quiquadrado=139,59; $p=0,001$ ). A comparação não-paramétrica dos escores da escala Blessed entre as categorias da CDR mostrou diferença significativa do ranking das medianas entre todas as categorias (Kruskal-Wallis, qui-quadrado=226,26; $P=0,001$ ).

A distribuição das categorias da escala CDR pelas 4 categorias do MEEM estão apresentadas na Tabela 3 (qui-quadrado=146,9; $p=0,001$; e razão de probabilidade=161,66; $p=0,001)$. A análise de concordância entre CDR e as categorias do MEEM mostrou um coeficiente kappa $=0,53(p=0,001)$ (Kendall's tau$b=0,61 ; p=0,001)$. Observou-se correlação significativa entre a escala CDR com idade (Spearman $r=0,17$; $p=0,008$ ), MEEM (Spearman $r=-0,72 ; p=0,001$ ), e Blessed (Spearman $r=0,96 ; p=0,001$ ). Não se observou 
Tabela 5. Testes cognitivos nos grupos diagnósticos (de acordo com a seleção para o estudo) e no grupo 0,5 da escala CDR.

\begin{tabular}{|c|c|c|c|}
\hline & $\begin{array}{c}\text { Demência } \\
\text { Questionável } \\
(\mathrm{N}=35)\end{array}$ & $\begin{array}{c}\text { CDR } 0,5 \\
(\mathrm{~N}=55)\end{array}$ & $\begin{array}{c}\text { Demência } \\
(\mathrm{DA}+\mathrm{DV}) \\
(\mathrm{N}=234)\end{array}$ \\
\hline \multicolumn{4}{|c|}{ Testes Cognitivos (média \pm DP) } \\
\hline MEEM & $23,13 \pm 3,95$ & $22,20 \pm 4,71$ & $16,74 \quad 6,94$ \\
\hline Extensão de dígitos & $4,60 \pm 1,20$ & $4,73 \pm 1,54$ & $3,80 \quad 2,04$ \\
\hline Extensão de palavras & $4,10 \pm 1,80$ & $3,56 \pm 1,65$ & $2,76 \quad 1,90$ \\
\hline Extensão de RV & $6,70 \pm 4,10$ & $8,6 \pm 3,4$ & $3,883,00$ \\
\hline MLI & $3,70 \pm 1,85$ & $3,00 \pm 1,53$ & $1,93 \quad 1,50$ \\
\hline MLII & $1,58 \pm 1,05$ & $1,2 \pm 1,41$ & $0,850,65$ \\
\hline Faces famosas & $11,30 \pm 2,07$ & $12,5 \pm 1,44$ & $6,25 \quad 5,03$ \\
\hline \multicolumn{4}{|l|}{$\%$ de alterados } \\
\hline Relógio & $28 \%$ & $20 \%$ & $44 \%$ \\
\hline Casa & $28 \%$ & $20 \%$ & $44 \%$ \\
\hline Cálculo & $50 \%$ & $60 \%$ & $83,5 \%$ \\
\hline Abstração & $60 \%$ & $50 \%$ & $82,5 \%$ \\
\hline Praxia & $3 \%$ & $0 \%$ & $20 \%$ \\
\hline
\end{tabular}

$\mathrm{RV}$, reconhecimento visual; MLI, memória lógica escore imediato; MLII, memória lógica escore tardio; DA, Doença de Alzheimer; DV, Demência vascular.

dife rença de escolaridade $(p=0,726)$ ou de idade $(p=$ $0,064)$ entre as categorias da escala CDR por ANOVA de 1-via (Tukey post hoc) (Tabela 4).

A Tabela 5 mostra o desempenho na bateria de testes cognitivos dos pacientes categorizados como demência questionável, demência (DA e vascular) de acordo com os critérios de entrada no estudo (padrão-ouro) e o grupo posteriormente identificado como "suspeitos" pela aplicação da escala de avaliação clínica da demência (CDR 0,5).

\section{DISCUSSÃO}

Com este estudo procuramos avaliar a concordância da escala CDR com os critérios diagnósticos provável demência vascular e DA e com o MEEM. A concordância com os critérios diagnósticos foi boa (kappa $=0,73)$, e moderada com o MEEM (kappa $=0,53)$. A avaliação de confiabilidade com investigadores de um estudo multicêntrico em DA usando treinamento, protocolo padrão e avaliações em vídeo de pacientes em vários estágios da DA, mostrou confiabilidade global de $83 \%{ }^{18}$. Num estudo populacional realizado em Estocolmo, Suécia, 224 casos de demência foram avaliados para gravidade usando duas escalas, CDR e DSM III-R ${ }^{19}$. Ambas escalas tiveram que ser modificadas para este objetivo e ao DSM III-R foi necessário a c rescentar a categoria demência questionável. Boa concordância foi observada quando todas as gravidades foram avaliadas (kappa $=0,60)$, mas moderada para as categorias questionável e leve (kappa $=0,47$ ).

A taxa de identificação de casos questionáveis em nosso estudo foi um pouco maior com a escala CDR do que com os critérios de inclusão utilizados no estudo para seleção dos pacientes, sugerindo que houve também mais flexibilidade na categorização de casos leves e menor concordância, similar ao que tem sido observado em outros estudos ${ }^{6,19}$. O estudo de Helsinki também mostrou concordância apenas moderada com o MEEM categorizado. Outros estudos têm mostrado concordâncias moderadas e baixas entre MEEM e CDR (kappa=0,33) ${ }^{20}$.

Um aspecto importante a ser ressaltado foi não te rmos observado diferença de escolaridade dos pacientes classificados nas diferentes categorias da CDR, sugerindo menor impacto da escolaridade sobre este instrumento. $\mathrm{O}$ fato da escala CDR ser mais abrangente e utilizar diversos aspectos funcionais, usar informação do familiar/cuidador bem como do próprio 
paciente pode ser a razão para a perda de influência da escolaridade.

Correlação com a escala de Blessed para gravidade foi observada mostrando que a escala CDR apresenta validade de conceito. A tentativa de obter instrumentos padronizados e validados no cenário brasileiro é im po rtante para auxiliar também na criação de consensos para centros que desenvolvem atividades de pesquisa e assistenciais com idosos e pacientes com demência. Um estudo publicado em 2005 mostrou que de diversos centros de DA nos países europeus, as escalas $C D R, A D L / I A D L$ e GDS eram as mais comumente empregadas (40-50\%), havendo diversidade indesejável no uso de instrumentos ${ }^{21}$. Os autores sugerem que um consenso deveria ser buscado.

Com um treinamento simples e com a utilização de rotina, a escala CDR pode ser utilizada como inst rumento de diagnóstico e classificação de gravidade de demência, pode ser utilizada para detecção de comprometimento cognitivo leve e para exclusão dos anterioresnum ambiente ambulatorial e mesmo em estudos de comunidades através da entrevista semiestruturada e entrevistadore adequadamente tre inados por ser de simples aplicação.

\section{REFERÊNCIAS}

1. Hughes CP, Berg L, Danziger WL, et al. A new clinical scale for the staging of dementia. Br J Psychiatry 1982;140:566-572.

2. Blessed G, Tomlinson BE, Roth $\mathrm{M}$. The association between quantitative measures of dementia and senile change in the cerebral gray matter of elderly subjects. Br J Psychiatry 1968;14:797-811.

3. Folstein MF, Folstein SE, McHugh PR. "Mini-mental state": a practical method for grading the cognitive state of patients for the clinician. J Psychiatr Res 1975;12:189-198.

4. http://alzheimer.wustl.edu/adrc2/Education/CDR\%20Inter-Page.html

5. McCulla MM, Coats M, Van Fleet N, Duchek J, Grant E, Morris JC. Reliability of clinical nurse specialists in the staging of dementia. Arch Neurol 1989; 46:1210-1211.
6. Tractenberg RE, Schafer K, Morris JC. Interobserver disagreements on Clinical Dementia Rating Assessment: interpretation and implications for Training. Alzheimer Dis Assoc Disord 2001;15:155-161.

7. Rockwood K, Strang D, MacKnight C, et. al. Interrater reliability of the Clinical Dementia Rating in a multicenter trial. J Am Geriatr Soc 2000;48:558-559.

8. Schafer KA, TractenbergRE, Sano M, et al. Reliability of monitoring the clinical dementia rating in multicenter clinical trials. Alzheimer Dis Assoc Disord 2004;18:219-222.

9. http: / / alzheimer.wustl.edu / adrc2 / Education / CDR / downloadselectionpage.htm

10. Beltrame A, Picon PD (eds). Protocolos clínicos e diretrizes terapêuticas: medicamentos excepcionais. Brasília Ministério Saúde, 2002:1-601.

11. American Psychiatric Association. Diagnostics and statistical manual of mental disorders, 4 th ed. Washington, DC: American Psychiatric Association; 1994.

12. McKhann G, Drachman D, Folstein M, et al. Clinical diagnosis of Alzheimer's disease: report of the NINCDS-ADRDAWork Group under the auspices of the Department of Health and Human Services Task Force on Alzheimer's Disease. Neurology 1984;34:939-944.

13. Roman GC, Tatemichi TK, Erkinjuntti T, et al. Vascular dementia: diagnostic criteria for researdhstudies. Report of the NINDS-AIREN International Workshop. Neurology 1993;43:250-260.

14. Hachinski VC, Miff LD, Zilkha E, et al. Cerebral blood flow in dementia. Arch Neurol 1975;32:632-637.

15. Katz S, Ford AB, Moskowitz RW, et al. The index of ADL: a st an d a rdized measure of biological and psychosocial function. JAMA 1963;185:914-919.

16. Chaves ML, Izquierdo I. Diffe rential diagnosis between dementia and depression: a study of efficiency increment. Acta Neurol Scand 1992;85:378-382.

17. Morris JC. The Clinical Dementia Rating (CDR): current version and scoring rules. Neurology 1993;43:2412-2414.

18. Morris JC. Clinical Dementia Rating: a reliable and valid diagnostic and staging measure for Dementia of the Alzheimer type. Int Psychogeriatr 1997;9(S1):S173-S176.

19. Forsell Y, Fratiglioni L, Grut M, et al. Clinical staging of dementia in a population survey: comparison of DSM III-R and theWashington University Clinical Dementia Rating Scale. Acta Psychiatr Scand 1992;86:49-54.

20. Juva K, Sulkava R, Erkinjuntti T, et al. Staging the severity of dementia: comparison of clinical (CDR, DSM III-R), functional (ADL, IADL) and cognitive (MMSE) scales. Acta Neurol Scand 1994;90:293-298.

21. Ramirez Diaz S, Gil Gregorio P, Manuel Ribra Casado J, et al. The need for a consensus in the use of assessment tools for Alzheimer's disease: the Feasibility Study (assessment tools for dementia in Alzheimer Centresacross Europe), a European Alzheimer's Disease Consortium's (EADC) survey. Int J Geriatr Psychiatry 2005;20:744-748. 